\title{
RFFECTIVE INTERACTION FOR PION ABSORPTION AND PRODUCTION BY TWO NUCLEONS AT LOW ENERGIES
}

\author{
I1-Tong CHEON* and J. CUGNON* \\ Institut de Physique, Université de Liège, Sart Tilman, \\ B-4000 Liege 1, Belgium
}

Received 27 December 1972

(Revised 2 April 1973)

Abstract: An effective interaction which can be applied to the problems of pion absorption and production at low energy is proposed in an elegant form. This effective interaction includes the pion rescattering effects which are very important in the absorption and production processes. The interaction proposed here is examined under consideration of the Coulomb effects in the process $\pi^{+}+d \rightarrow p+p$ at very low energies. The determination of the scattering lengths is attempted. For $a_{1}-a_{3}=0.291$ the best fit is $a_{1}-a_{3}=-0.055$. The absorption rate of a pion by a deuteron from the atomic $1 S$ state is also calculated. The result is $W=1.10 \times 10^{16} \mathrm{see}^{-1}$. It should be stressed that our theory includes no free parameter.

\section{Introduction}

Pion absorption is accepted to be a good tool for the investigation of various properties of light nuclei [1] such as short-range NN correlation [2], two hole excitations, model wave function and so on. The earlier investigations of these problems were made with the simple interaction which was constructed on the basis of the usual psetidoscalar $\pi \mathrm{N}$ interaction. Moreover, most of them did not include the rescattering effects. Here, the rescattering effects in pion absorption mean the process by which the first nucleon emits a pion after or before absorbing the pion from outside and the second nucleon absorbs the pion emitted by the first nucleon. It is surprising that some people are treating these processes with the crude interaction mentioned above even after the importance of the rescattering was pointed out $[3,4]$.

Koltun and Reitan [3] discussed the rescattering effects on the reactions $p+p \rightarrow d+\pi^{+}$and $p+p \rightarrow p+p+\pi^{0}$, by using the Hamiltonian which consists of the usual $p$-wave interaction, the Gallean invariant s-wave recoil term, the meson

\footnotetext{
* Chercheur I, S.S.N.
} 
pair term and the isotopic-spin dependent quadratic s-wave interaction. The last two terms can be obtained as an approximation to the pseudoscalar interaction by a Foldy Dyson transformation [5]. The same Hamiltonian was previously used by Woodruff $[6]$ in the analysis of the process $p+p \rightarrow d+\pi^{+}$near threshold. The results obtained by Koltun and Reitan are in agreement with the measurements of Field and al. [7], but disagree with the recent measurements (see our fig. 3). However, in this approach, it is not possible to classify the time ordering of the pion absorption and scattering by a nucleon. Therefore, the Feynman graphs which should be included in the final state interaction or the nuclear correlation of the initial state of two nucleons are also counted in the calculation of the matrix elements. These graphs should be removed, otherwise one makes a mistake by overestimating the matrix elements. The Hamiltonian used by Koltun et al. [3] and Woodruff [6] is not convenient to do this. Actually, it is impossible. Furthermore, since their theory is restricted to s-wave pion production, it is useless for the processes by which the pions are absorbed by nuclei from the atomic $2 \mathrm{P}$-orbit. Moreover, Coulomb effects are neglected.

Cheon and Nishimoto et al. discussed the process $\pi^{+}+d \rightarrow p+p$ in the frame of $S$-matrix theory [4]. However, since the treatment of the energy denominators in the matrix elements was not reasonable in our previous paper, good agreement with experimental data could not be obtained without introducing a free parameter into the coupling constant. It should be noticed that the same approach was applied by Nishimoto et al. to the problems of pionic atoms [4].

Recently, Lazard et al. [8] calculated the process $\pi^{+}+d \rightarrow p+p$ from threshold to $300 \mathrm{MeV}$ pion kinetic energy. Their data is essentially the same as Cheon's [4] in the sense that for the $\pi \mathrm{N}$ scattering vertex the parametrized scattering amplitude was used instead of the usual Hamiltonian formalism, but they made use of the wellknown $\pi \mathrm{N}$ scattering amplitude proposed by Chew et al. [9]. They obtained good results for the total cross section and the angular distributions. As was pointed out by Richard-Serre et al. [10] their calculation is questionable in the high energy regions because their work is non-relativistic. Even in the low energy region, their calculation is not consistent in the following points. For two protons in the final state they used the asymptotic wave function whose short-range part was essentially. described by a phenomenological cut-off function. A parameter was included in the cut-off function and was determined by fitting the experimental data. This is not a satisfactory procedure, since, as pointed out by them, the cross sections are very sensitive to the behavior of the wave function in the short-range region. It is highly desirable to use as wave functions, for the two outgoing protons and for the deuteron, those obtained by solving the Schrödinger equation with the realistic NN potential. Accordingly, the work of Lazard et al. is not complete.

The work of Schiff and Tran [11] on the pion production is important in the sense that it is the first completely relativistic calculation without free parameters. Unfortunately, they neglected the s-wave production and only the p-wave was taken into account. 
In the present paper, we remove those defects mentioned above and derive a more elegant form of the $\pi \mathrm{NN}$ effective interaction without free parameters. Our theoretical motivation is the same as in the previous paper [4], namely derivation of the effective $\pi \mathrm{NN}$ Hamiltonian which can be used for the calculation of the stopped $\pi^{-}$meson absorption by nuclei. Therefore, our theory will be developed in the non-relativistic frame.

As applications of our effective interaction, we consider the reactions $\pi^{+}+\mathrm{d} \rightarrow \mathrm{p}+\mathrm{p}$ at very low energy and also the $\pi^{-}$meson absorption by a deuteron from the atomic $1 \mathrm{~S}$ state. A discussion is given in the last section on the $\pi \mathrm{N}$ scattering length $a_{1}+2 a_{3}$ which is left unsatisfactorily determined, although a lot of calculations have been done.

\section{Derivation of the $\pi \mathbb{N N}$ effective interaction}

In this section, we shall treat the process in which a pion is absorbed by two nucleons. We use the units $\hbar=c=1$ unless indications are specially given. Our task is to calculate the matrix elements according to the graphs given in fig. 1. Fig. 1a corresponds to the direct absorption term and figs. $1 \mathrm{~b}$ and $1 \mathrm{c}$ describe the pion rescattering by the nucleon 1 forward and backward in time, respectively. The graphs which we must calculate here are only crossing diagrams since other graphs are included in the initial state NN correlation and the final state interactions. The shaded area which is called a scattering part contains a number of virtual pions emitted and adsorbed by a nucleon. This scattering part is different from the usual $S$-matrix ele-

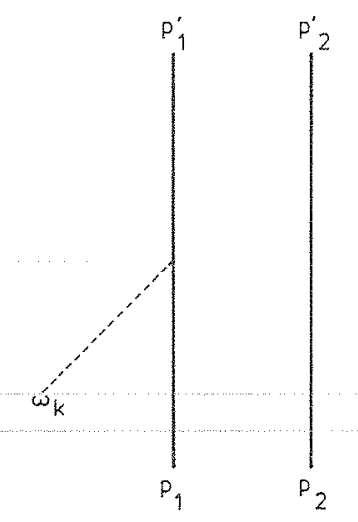

(a)

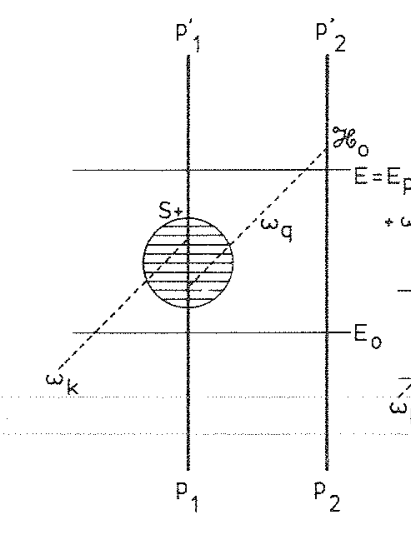

(b)

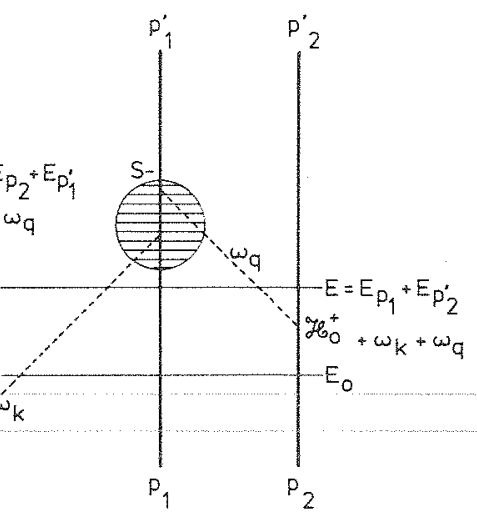

(c)

Fig. 1. Feynman graphs for the process $\pi+N+N \rightarrow N+N$. (a) Direct absorption. (b) A pion is rescattered forward in time. (c) A pion is rescattered backward in time. 
ment in the sense that the pions are virtual in the shaded area and do not satisfy the energy relation $k_{0}^{2}=k^{2}+\mu^{2}$ as real pions do. The generalized $S$-matrix which contains a scattering part was first used by Miyazawa [12] in a calculation of the proton anomalous magnetic moment.

\subsection{Definition of the $\pi$-nucleon interaction}

The pion-nucleon interaction densities are given in the forms

$$
\begin{aligned}
& S_{+}^{\mathrm{p}}(l)=4 \pi i \delta\left(k_{0}-q_{0}\right) \tilde{A}\left(k_{0}\right) \tau_{i} \tau_{j}(\sigma \cdot k)(\sigma \cdot q) \mathrm{e}^{i(k-q) \cdot r_{l}}, \\
& S_{-}^{\mathrm{p}}(l)=4 \pi i \delta\left(k_{0}-q_{0}\right) \tilde{C}\left(k_{0}\right) \tau_{j} \tau_{i}(\sigma \cdot q)(\sigma \cdot k) \mathrm{e}^{i(k+q) \cdot r_{l}}, \\
& \mathscr{H}_{0}^{\mathrm{p}}(l)=\sqrt{4 \pi} \frac{i f}{\mu}\left(\sigma \cdot \nabla_{l}^{\pi}\right)(\tau \cdot \tilde{\phi}), \\
& \mathscr{L}_{0}^{\mathrm{p}}(l)=\sqrt{4 \pi} \frac{i f}{\mu}\left(\sigma \cdot \nabla_{l}^{\pi}\right)\left(\tau \cdot \tilde{\phi}^{\dagger}\right),
\end{aligned}
$$

for a $\mathrm{p}$-wave pion and

$$
\begin{aligned}
& S_{+}^{s}(l)=4 \pi i \delta\left(k_{0}-q_{0}\right) D\left(k_{0}\right) \tau_{i j} \mathrm{e}^{i(k-q) \cdot \hat{p}_{l}}, \\
& S_{-}^{s}(l)=4 \pi i \delta\left(k_{0}-q_{0}\right) E\left(k_{0}\right) \tau_{i} \tau_{j} \mathrm{e}^{i(k+q) \cdot r_{l}}, \\
& x_{0}^{\mathrm{s}}(l)=-\sqrt{4 \pi} \frac{\text { if }}{M}\left(\sigma \cdot \nabla \frac{\mathrm{N}}{l}\right)(\tau \cdot \tilde{\phi}), \\
& \mathscr{x}_{0}^{\mathrm{s \dagger}}(l)=-\sqrt{4 \pi} \frac{i f}{M}\left(\sigma \cdot \nabla \frac{N}{l}\right)\left(\tau \cdot \widetilde{\phi}^{\dagger}\right)
\end{aligned}
$$

for a s-wave pion. Here $\mu$ and $M$ are the pion and nucleon masses, $\tau$ and $\sigma$ are the nuclear isospin and spin operators, $k$ ard $q$ are the momenta of the incoming and rescattered pion, $f$ is a renormalized coupling constant $f^{2}=g^{2} \bar{h} c\left(g^{2}=0.08\right)$, and $\nabla_{l}^{\pi}$ and $\nabla_{l}^{\mathrm{N}}$ are the operators acting on a pion and a nucleon at the position of the nucleon $\left.l: \widetilde{\phi}_{\dagger}+\widetilde{\phi}_{+}^{+}, \widetilde{\phi}_{0}^{\dagger}, \widetilde{\phi}_{-}^{\dagger}\right)$ and $\left.\widetilde{\phi}_{\left(\tilde{\phi}_{-}\right.}, \widetilde{\phi}_{0}, \widetilde{\phi}_{+}\right)$are creation and annihilation operators of $\pi^{+}, \pi^{0}, \pi^{-}$, respectively. The matrix elements of these operators between a pion state of momentum $q$ and the vacuum state are given by

$$
\begin{aligned}
& \left\langle q\left|\tilde{\phi}^{\dagger}\right| 0\right\rangle=\frac{1}{\sqrt{2 q_{0}}} \mathrm{e}^{-i q \cdot r}, \\
& \langle 0|\tilde{\phi}| q\rangle=\frac{1}{\sqrt{2 q_{0}}} \mathrm{e}^{i q \cdot r} .
\end{aligned}
$$


The quantities $\widetilde{A}, \widetilde{C}, D, E$ are given in ref. [12]. Let us give the explicit forms of $D$ and $E$, which will be useful later.

$$
\begin{aligned}
& D\left(k_{0}\right)=\frac{2 \pi}{3}\left(a_{1}+2 a_{3}\right)-\frac{2 \pi k_{0}}{3 \mu}\left(a_{1}-a_{3}\right)+\frac{k_{0}^{2}-\mu^{2}}{2 \pi} \int_{0}^{\infty} \frac{\mathrm{d} p}{\omega_{p}} \\
& \times\left[\frac{\sigma_{3}}{\omega_{p}-k_{0}-i \epsilon}+\frac{\sigma_{3}+2 \sigma_{1}}{3\left(\omega_{p}+k_{0}-i \epsilon\right)}\right], \\
& E\left(k_{0}\right)=D\left(-k_{0}\right) .
\end{aligned}
$$

The interaction densities (3) and (4) come from the non-relativistic limit of the pseudoscalar $\pi \mathrm{N}$ interaction. The interaction densities (7) and (8) are called Galilean invariance terms. $S_{+}$and $S_{-}$are the $S$-matrices corresponding to forward and backward scattering in time, respectively (see fig. 1).

\subsection{Construction of the S-matrix}

In order to construct the $S$-matrix describing the pion absorption by a nucleon pair with the rescattering effects using the interaction desities (1) to (8), we must calculate the energy denominator. In fig. (1b), the energy in the intermediate state is

$$
E=E_{p_{2}^{\prime}}+E_{p_{1}^{\prime}}+w_{q}
$$

and the total energy of the initial state is

$$
E_{0}=\omega_{k}+E_{p_{1}}+E_{p_{2}}
$$

where $E_{p_{1}}, E_{p_{2}}, E_{p_{1}^{\prime}}$ and $E_{p_{2}^{\prime}}$ correspond, respectively, to the energies of the nucleons 1 and $2_{2}$ in the initial and final states, and $\omega_{k}$ and $\omega_{q}$ are the energies of the incoming and rescattered pions. Notice that $E$ is not equal to $E_{0}$ since $q_{0}$ does not satisfy the mass formula $q^{2}+\mu^{2}$; however $E_{1}=E_{p_{1}^{\prime}}+E_{p_{2}}+q_{0}$ is equal to $E_{0}$ by the energy conservation law. When the nucleons 1 and 2 share the energy equally between each other, namely $E_{p_{1}}=E_{p_{2}}$ and $E_{p_{1}^{\prime}}=E_{p_{2}^{\prime}}$, we have

$$
\Delta E=E-E_{0}=\omega_{q}-\frac{1}{2} \omega_{k}
$$

Similarly, we have

$$
\Delta E=\omega_{q}+\frac{1}{2} \omega_{k}
$$


for the graph (c) in fig. 1. Therefore, if we put $q_{0}=\frac{1}{2} \omega_{k}$ in the usual form of the denominator $q_{\mu}^{2}$ in the four momentum space, we can obtain the correct energy denominator.

Thus, the $S$-matrix for the process considered here is given as

$$
\begin{aligned}
& \langle\mathrm{NN}|S| \mathrm{NN} \pi\rangle=\sum_{l \neq m=1}^{2} \frac{i}{(2 \pi)^{4}} \int \frac{\mathrm{d}^{4} q \delta\left(q_{0}-\frac{1}{2} \omega_{k}\right)}{q_{0}^{2}-\omega_{q}^{2}+i \epsilon} \\
& \times\left[\left\langle 0\left|\mathscr{X}_{0}(l)\right| q\right\rangle\left\langle q\left|S_{+}(m)\right| k\right\rangle+\left\langle 0\left|S_{-}(l)\right| q, k\right\rangle\left\langle q\left|q_{0}^{\dagger}(m)\right| 0\right\rangle\right] .
\end{aligned}
$$

The first and second terms correspond to the forward (graph b) and backward (graph c) scattering in time, respectively. As long as the two nucleon system is antisymmetrized, the exchange of the particles 1 and 2 in fig. 1 makes no difference, namely the same matrix element comes from it.

\subsection{Effective $\pi N N$ interaction}

In the way described in [4], we are able to construct the effective interactions for the pion absorption or production by the two nucleon system. We summarize

$$
\begin{aligned}
& H_{0}^{\mathrm{s}}=-\frac{(-)^{i} \hbar c}{\sqrt{2 \omega_{k}}} \frac{\sqrt{4 \pi} f h}{M c}\left(T_{i} R_{1}^{\mathrm{N}}+\tau_{i} R_{2}^{M}\right) \hat{\phi} \\
& H^{s s}=-\frac{(-)^{i}{ }^{n} c}{\sqrt{2 \omega_{k}}} \frac{i \sqrt{2}}{\sqrt{\pi}} \frac{f n}{M c}\left[D\left(\frac{\omega_{k}}{2}\right)\left(R_{1}^{N} T_{i}-R_{2}^{N} \tau_{i}\right) y\right. \\
& +E\left(\frac{\omega_{k}}{2}\right) J\left(T R_{1}^{\mathbb{N}}-\tau_{i} R_{2}^{N}\right) \frac{e^{-\beta r}}{r} \hat{\phi}, \\
& H^{\mathrm{sp}}=\frac{(-)^{i} \hbar c}{\sqrt{2 \omega_{k}}} \frac{i \sqrt{8}}{\sqrt{\pi}} \frac{f n}{\mu c}\left[D\left(\frac{\omega_{k}}{2}\right)\left(R_{3} T_{i}-R_{4} \tau_{i}\right) J\right. \\
& +E\left(\frac{\omega_{k}}{2}\right) J\left(T_{i} R_{3}-\tau_{i} R_{4}\right) \frac{e^{-\beta r}}{r} \hat{\phi},
\end{aligned}
$$

for the s-wave pion, and

$$
H_{0}^{\mathrm{p}}=\frac{(-)^{i} \hbar c}{\sqrt{2 \omega_{k}}} \frac{\sqrt{4 \pi} f h}{\mu c}\left(T_{i} R_{1}^{\pi}+\tau_{i} R_{2}^{\pi}\right) \hat{\phi}
$$




$$
\begin{aligned}
H^{\mathrm{pp}} & =-\frac{(-)^{i} \hbar c}{\sqrt{2 \omega_{k}}} \frac{i \sqrt{2}}{\sqrt{\pi}} \frac{f \hbar}{\mu c}\left[\widetilde{A}\left(\frac{\omega_{k}}{2}\right)\left(T_{i} R_{1}^{\pi}-\tau_{i} R_{2}^{\pi}\right) J Z_{12}(r)\right. \\
\left.-\widetilde{C}\left(-\frac{\omega_{k}}{2}\right) Z_{12}(r) J\left(T_{i} R_{1}^{\pi}+\tau_{i} R_{2}^{\pi}\right)\right] \hat{\phi} & \\
H^{\mathrm{ps}} & =-\frac{(-)^{i} \hbar c}{\sqrt{2 \omega_{k}}} \frac{\sqrt{2}}{2 \sqrt{\pi}} \frac{f \hbar}{M c}\left[\widetilde{A}\left(\frac{\omega_{k}}{2}\right)\left(R_{11}^{\mathrm{N} \pi} R_{3}+R_{12}^{N \pi} R_{4}\right) T_{i} J\right. \\
& \left.+\left(R_{12}^{\mathrm{N} \pi} R_{3}+R_{11}^{\mathrm{N} \pi} R_{11}^{\mathrm{N} \pi} R_{4}\right) \tau_{i} J\right) \\
& -\widetilde{C}\left(-\frac{\omega_{k}}{2}\right)\left(\left(R_{3} R_{11}^{\pi \mathrm{N}}-R_{4} R_{12}^{\pi \mathrm{N}}\right) J T_{i}\right. \\
& \left.\left.-\left(R_{3} R_{12}^{\pi \mathrm{N}}-R_{4} R_{11}^{\pi \mathrm{N}}\right) J \tau_{i}\right)\right] \frac{\mathrm{e}^{-\beta r}}{r} \hat{\phi}
\end{aligned}
$$

for the p-wave pion, where

$$
\begin{aligned}
& R_{1}^{\mathrm{N}(\pi)}=S \cdot \mathbb{P}_{R}^{\mathrm{N}(\pi)}+2 \sigma \cdot \nabla_{r}^{\mathrm{N}(\pi)}, R_{2}^{\mathrm{N}(\pi)}=\sigma \cdot \nabla_{R}^{\mathrm{N}(\pi)}+2 S \cdot \nabla_{r}^{\mathrm{N}(\pi)}, \\
& R_{3}=\sigma \cdot \nabla_{r}, \quad R_{4}=S_{r} \cdot \nabla_{r}, \\
& R_{1 j}^{\mathrm{N} \pi}=R_{1}^{\mathrm{N}} R_{j}^{\pi}-R_{2}^{\mathrm{N}} R_{3-j}^{\pi}, \quad R_{1 j}^{\pi \mathrm{N}}=R_{1}^{\pi} R_{j}^{\mathrm{N}}-R_{2}^{\pi} R_{3-j}^{\mathrm{N}}, \quad j=1,2 .
\end{aligned}
$$

The indices $\mathrm{N}$ and $\pi$ denote the operators acting on the nuclear and pion states. $R_{3}$ and $R_{4}$ operate on the virtual pion state $\mathrm{e}^{-\beta r} / r$. The quantities $\beta$ and $Z_{12}(r)$ are given as

$$
\beta^{2}=\mu^{2}-\frac{1}{4} \omega_{k}^{2}
$$

and

$$
Z_{12}(r)=\left(\sigma_{1} \cdot \nabla_{r}\right)\left(\sigma_{2} \cdot \nabla_{r}\right) \frac{\mathrm{e}^{-\beta r}}{r}=\left[\frac{\beta^{2}}{3}\left(\sigma_{1} \cdot \sigma_{2}\right)+\left(\frac{1}{r^{2}}+\frac{\beta}{r}+\frac{\beta^{2}}{3}\right)^{S_{12}}\right] \frac{\mathrm{e}^{-\beta r}}{r}
$$

where $S_{12}$ is the tensor operator

$$
S_{12}=\frac{3}{r}\left(\sigma_{1} \cdot r\right)\left(\sigma_{2} \cdot r\right)-\left(\sigma_{1} \cdot \sigma_{2}\right)
$$


For the isospin state of the two nucleon system $X_{\tau \lambda}$, we have

$$
J X_{\tau \lambda}=\left(1+4 \lambda^{2}-4 \tau\right) X_{\tau \lambda}
$$

The interactions (17) and (20) correspond to the direct processes in which the pion is directly absorbed or produced by either nucleon without exchange of any pion. The pion field operator with a hat is given in the form [4]

$$
\left\langle 0|\hat{\phi}| k_{0}\right\rangle=\left(\mathrm{e}^{i k_{0} \circ r_{1}}+\mathrm{e}^{i k_{0} \circ r_{2}}\right) / 2 \sqrt{V}=\sqrt{\frac{A \pi}{V}} \mathrm{e}^{i k_{0} \cdot R}\left[\dot{j}_{0}\left(\frac{k_{0}}{2} r\right) Y_{00}(\hat{r})+\ldots\right] .
$$

In the case of the bound pion, it should be replaced by a bound state wave function. For example, for the pion in the $1 \mathrm{~S}$ atomic orbit

$$
\langle 0|\hat{\phi}| 1\rangle=\frac{2 z^{\frac{3}{2}}}{a_{0}^{\frac{3}{2}}} \exp \left(-\frac{r}{2 a_{0}}\right) Y_{00}(\hat{r})
$$

under the assumption of a point charge. The quantity $a_{0}$ is the Bohr radius.

Here, we should stress that our effective $\pi N N$ interactions do not include any free parameter and are different in content from the previous papers $[3,4]$. These changes in the contents give better results for analysis of $\pi^{+}+d \leftrightharpoons p+p$ and $p+p \rightarrow p+p+\pi^{0}$.

\section{Analysis of the process $\pi^{+}+\tilde{d} \rightarrow p+p$ at low energies}

It is very interesting to examine our effective interaction in the analysis of the process $\pi^{+}+d \rightarrow p+p$ at low energies where the $S$-wave contribution is dominant. Rose [13] performed the experiment in the energy range $2-16 \mathrm{MeV}$, even though the experiment is very difficult in this energy region. The results obtained by Rose are shown in fig. 2 . The phenomenologically fitted curve for his experiment alone was given by [13]

$$
\sigma\left(\pi^{+}+d \rightarrow p+p\right)=\frac{2}{3} \frac{p_{p}^{2}}{\mu c^{2}}\left[C_{1}^{2}(\eta) \frac{\alpha}{\eta}+C_{2}^{2}(\eta) \beta \eta\right]
$$

where

$$
\alpha=0.240 \mathrm{mb} \pm 0.02 \mathrm{mb}, \quad \beta=0.52 \mathrm{mb} \pm 0.2 \mathrm{mb}, \quad \eta=\frac{p_{\pi}\left(\mathrm{cm} . \mathrm{m}_{0}\right)}{\mu \mathrm{c}}
$$




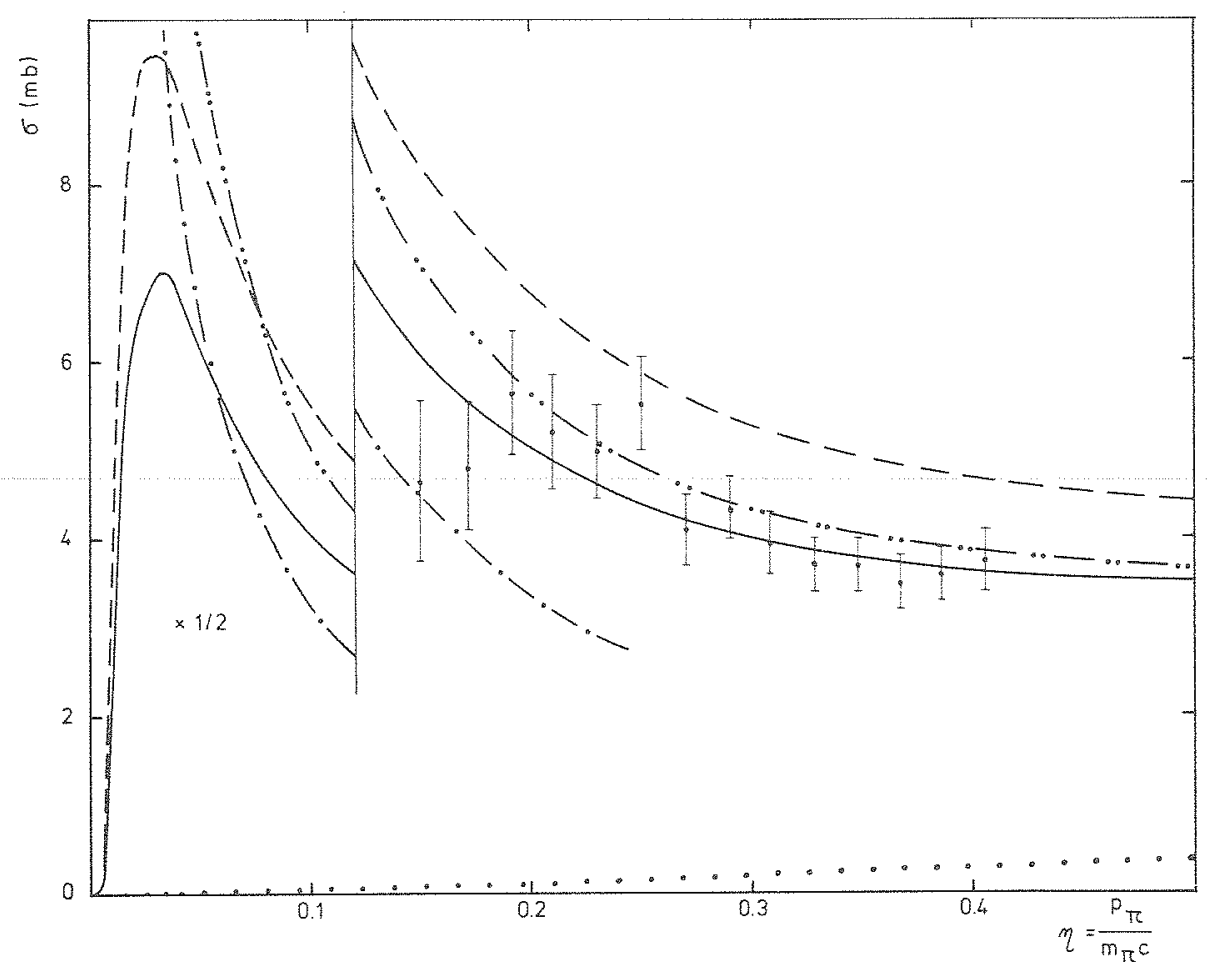

Fig. 2. The total cross sections for the process $\pi^{+}+d \rightarrow p+p$. The solid line corresponds to $\lambda=0.251 \mathrm{fm}$ and the dashed line was obtained with $\lambda=0.31 \mathrm{~m}$. The $\mathrm{p}$-wave contribution is taken into account by the second term of eq. (29). The dot-dot-dashed line corresponds to the result with $\lambda=0.251 \mathrm{~m}$ and without Coulomb effects. The dotted line is obtained with $\lambda=0$ (this curve is exaggerated by multiplication of a factor 62 ). The dot-dashed line is the result derived from the Koltun-Reitan calculation.

These values were obtained with a $\chi^{2}$ of 13.4 for 16 degrees of freedom. $C_{1}(\eta)$ and $C_{2}(\eta)$ are the Coulomb correction factors.

Koltun and Reitan [3] calculated the total cross section of the reaction $p+p \rightarrow d+\pi^{+}$at threshold energy on the basis of the phenomenological S-wave interaction which was obtained by a Foldy-Dyson transformation [5]. The total cross section of the inverse process $\pi^{+}+\mathrm{d} \rightarrow \mathrm{p}+\mathrm{p}$ can be obtained by detailed balance. However, their theory cannot give good agreement with the new experimental data produced by Rose.

\subsection{Derivation of the total cross section}

We take the deuteron wave function and the final two outgoing protons state to be given as follows: 


$$
\begin{aligned}
& \left.\left.|I\rangle=(2 \pi)^{-\frac{3}{2}} V^{-\frac{1}{2}} e^{i K \cdot R} \frac{1}{r}\left[\left.u(r)\right|^{3} S_{1}\right\rangle+\left.w(r)\right|^{3} D_{1}\right\rangle\right] \\
& |F\rangle=(2 \pi)^{-\frac{3}{2}} V^{-1} e^{i K^{\prime} \cdot R} 4 \pi \sum i^{l^{\prime}}\left(l^{\prime} m^{\prime} S^{\prime} v^{\prime} \mid J^{\prime} M^{\prime}\right) \\
& \times Y_{l^{\prime} n^{\prime}},(\hat{k}) Y_{l^{\prime} m^{\prime}}^{*}(\hat{\gamma}) g_{l^{\prime} J^{\prime}}(k r) \chi_{s^{\prime} \nu^{\prime}} \chi_{T^{\prime} \Lambda^{\prime}}
\end{aligned}
$$

For the moment we neglect the Coulomb effects which will be discussed later. Here $K$ and $K^{\prime}$ are the wave vectors of the initial and final state, and $R$ is the position vector of the center-of-mass of the two nucleon system. The functions $\chi_{S^{\prime} \nu^{\prime}}$ and $\chi_{T^{\prime \prime} \Lambda^{\prime}}$ are the spin and isospin states of the two proton system, and $V$ is the normalization volume, We used the symbols $S^{\prime}, T^{\prime}, l^{\prime}$ and $I^{\prime}$ for the spin, isospin, orbital angular momentum and the total angular momentum of the final state. The function $g_{l^{\prime} J^{\prime}}^{S^{\prime} T^{\prime}}(\mathrm{kr})$ is the solution (in the scattering state) of the Schrödinger equation with the nucleon-nucleon potential.

Since the transition ${ }^{3} S_{1} \rightarrow{ }^{3} P_{1}$ is allowed for the $S$-wave pion absorption by a deuteron by the selection rule, we have actually $S^{\prime}=1, T^{\prime}=1, l^{\prime}=1$ and $J^{\prime}=1$. The wave function of the pion has been already given in eq. (27). For the $S$-wave pion considered here, we must cut off the higher partial waves in eq. (27), i.c. only the first term is actually acting.

The matrix elements of the effective interactions (17), (18) and (19) with respect to the initial and final states (31) and (32) are easily calculated. The absorption cross section is:

$$
\sigma\left(\pi_{\mathrm{s}}^{+}+\mathrm{d} \rightarrow \mathrm{p}+\mathrm{p}\right)=\frac{16 \pi}{3} g^{2} \frac{\hbar}{M c} \frac{k}{k_{0}}\left[F\left(k, k_{0}\right)\right]^{2},
$$

where

$$
\begin{aligned}
& F\left(k, k_{0}\right)=\left\{L_{0}^{\mathrm{S}}\left(k, k_{0}\right)+\frac{1}{\sqrt{2}} L_{0}^{\mathrm{D}}\left(k, k_{0}\right)\right\}+\lambda \sqrt{ } 2\left[L_{1}^{\mathrm{S}}\left(k, k_{0}\right)+\frac{1}{\sqrt{2}} L_{1}^{\mathrm{D}}\left(k, k_{0}\right)\right\} \\
& \left.\quad-\frac{M}{\mu}\left\{L_{2}^{\mathrm{S}}\left(k, k_{0}\right)+\frac{1}{\sqrt{2}} L_{2}^{\mathrm{D}}\left(k, k_{0}\right)\right]\right] \\
& \beta=-\left[D\left(\frac{1}{2} \omega_{k}\right)+E\left(-\frac{1}{2} \omega_{k}\right)\right] / 2 \pi, \\
& \beta=\frac{\sqrt{3}}{2} \frac{\mu c}{\hbar}, \\
& L_{0}^{\mathrm{S}}=\int_{0}^{\infty} g_{11}^{11}(k r) j_{0}\left(\frac{k_{0} r}{2}\right)\left(\frac{\mathrm{d}}{\mathrm{d} r} \frac{\mu(r)}{r}\right) r^{2} \mathrm{~d} r
\end{aligned}
$$




$$
\begin{aligned}
& L_{1}^{\mathrm{S}}=\int_{0}^{\infty} g_{11}^{11}(k r) j_{0}\left(\frac{k_{0} r}{2}\right) e^{-\beta r}\left(\frac{\mathrm{d}}{\mathrm{d} r} \frac{u(r)}{r}\right) r \mathrm{~d} r, \\
& L_{2}^{\mathrm{S}}=\int_{0}^{\infty} g_{11}^{11}(k r) j_{0}\left(\frac{k_{0} r}{2}\right)\left(\frac{\mathrm{d}}{\mathrm{d} r} \frac{\mathrm{e}^{-\beta r}}{r}\right) u(r) r \mathrm{~d} r, \\
& L_{0}^{\mathrm{D}}=\int_{0}^{\infty} g_{11}^{11}(k r) j_{0}\left(\frac{k_{0} r}{2}\right)\left(\left(\frac{\mathrm{d}}{\mathrm{d} r}+\frac{3}{r}\right) \frac{w(r)}{r}\right) r^{2} \mathrm{~d} r, \\
& L_{1}^{\mathrm{D}}=\int_{1}^{\infty} g_{11}^{11}(k r) j_{0}\left(\frac{k_{0} r}{r}\right) \mathrm{e}^{-\beta r}\left(\left(\frac{\mathrm{d}}{\mathrm{d} r}+\frac{3}{r}\right) \frac{w(r)}{r}\right) r \mathrm{~d} r, \\
& L_{2}^{\mathrm{D}}=\int_{0}^{\infty} g_{11}^{11}(k r) j_{0}\left(\frac{k_{0} r}{2}\right)\left(\frac{\mathrm{d}}{\mathrm{d} r} \frac{\mathrm{e}^{-\beta r}}{r}\right) w(r) r \mathrm{~d} r .
\end{aligned}
$$

From these equations, it can be seen that $F\left(k, k_{0}\right)$ is finite in the limit of $k_{0} \rightarrow 0$ (the kinetic energy of the pion is zero). $k$ has also a finite value in this limit. Therefore, the total cross section goes to infinity when $k_{0}$ goes to zero. This difficulty is caused by the neglect of the Coulomb effects. At low energies, these effects can be estimated by multiplying the cross section by the Coulomb factor. Thus, the total cross section becomes

$$
\sigma\left(\pi_{\mathrm{S}}^{+}+\mathrm{d} \rightarrow \mathrm{p}+\mathrm{p}\right)=\left|\mathrm{e}^{-\frac{1}{2} \pi \zeta} \Gamma(1+i \zeta)\right|^{2} \frac{16 \pi}{3} g^{2} \frac{\hbar}{M c} \frac{k}{k_{0}}\left[F\left(k, k_{0}\right)\right]^{2}
$$

in the c.m. system, where $\xi=M_{\mathrm{r}} e^{2} / \hbar q(q$ is the momentum of relative motion) with $M_{\mathrm{r}}^{-1}=M_{\mathrm{d}}^{-1}+\mu^{-1}$.

\subsection{Numerical results}

In the present calculation, we used the Hamada-Johnston potential in order to obtain the wave functions of the deuteron and of the two outgoing protons. The results are shown with the experimental points obtained by Rose [13] in fig. 2 . The dashed line was obtained under consideration of the Coulomb effects with $\lambda=0.31 \mathrm{fm}$. The contributions of the $\mathrm{p}$-wave pion were taken into account by the phenomenological formula, namely the second term of eq. (29). We emphasize the importance of retardation effects [14], which is the last term in the expression of $D$ (see eq. (11)). The solid line is obtained with the Coulomb effects when $\lambda=0.251$ $\mathrm{fm}$ is chosen. This value is determined by the least squares with a $\chi^{2}$ of 12.2 for 14 
degrees of freedom. Our solid line is very similar to the phenomenological one eq. (29) which was obtained by Rose [13]. In order to examine the Coulomb effects, we have also drawn with a dot-dot-dashed line the result in which the Coulomb ef. fects were completely neglected. This curve goes to infinity as the kinetic energy of incident pion goes to zero. This fact can easily be seen in eq. (33). Therefore, we conclude that the Coulomb effects become considerably important in the region below $\eta=p$ (c.m.)/ $\mu c=0.2$. For this value, the Coulomb correction is about $12 \%$. With the value $\lambda=0.232 \mathrm{fm}\left(a_{1}+2 a_{3}=-0.035, a_{1}-a_{3}=0.291\right)$ [15], we obtained almost the same result as Reitan's [16], which was laid in a slightly lower site than the solid line in Fig. 2. We should emphasize that only with the direct term of the effective $\pi \mathrm{NN}$ interaction, we cannot reproduce the theoretical total cross section which is in agreement with the experimental data for the process considered here. It is, however, difficult to see in fig. 2. the behavior of the rescattering effects at zero kinetic energy. This can be seen in the $\pi^{-}$absorption from an atomic orbit which will be discussed in the following section.

For comparison, the result obtained by Koltun and Reitan [3] is also shown with a dot-dashed line in fig. 2 . This was obtained by detailed balance

$$
\sigma\left(\pi^{+}+\mathrm{d} \rightarrow \mathrm{p}+\mathrm{p}\right)=\frac{2}{3} \frac{p_{\mathrm{p}}^{2}}{p_{\pi}^{2}} \sigma\left(\mathrm{p}+\mathrm{p} \rightarrow \pi^{+}+\mathrm{d}\right)
$$

since they actually calculated the process $p+p \rightarrow \pi^{+}+d$ at threshold. It is not suitable to extend this relation to large $p_{\pi}$ because their calculation is restricted near the threshold.

The contributions of each term in the effective $\pi N N$ interaction, (17), (18), (19), to the total cross section can be seen in table 1 in connection with eq. (34). The quantities $L_{0}\left(k, k_{0}\right), L_{1}\left(k, k_{0}\right)$ and $L_{2}\left(k, k_{0}\right)$ in eq. (38) are the contributions from $H_{0}^{\mathrm{s}}, H$ ss and $H^{\mathrm{sp}}$ in the effective $\pi \mathrm{NN}$ interaction, respectively. The superscripts $S$ and $D$ in eq. (34) and table 1 denote the contributions of the $S$ - and $D$ states of the deuteron wave function. The dominant contribution comes from the $H^{\mathrm{sp}}$ term. This can be seen in table 1 and eq. (34). $L_{0}^{\mathrm{S}}$ is opposite in sign to $L_{0}^{\mathrm{D}}$, and the values of $\left(L_{0}^{\mathrm{S}}+L_{0}^{\mathrm{D}} / \sqrt{ } 2\right)$ become very small. This is reflected in the fact that the contributions of the $H_{0}^{s}$ and $H^{s s}$ terms in the effective interaction are actually small. The circumstances are the same in the case of $L \mathrm{~S}_{1}^{\mathrm{S}}$ and $L{ }_{1}^{\mathrm{D}}$. On the other hand, $L_{2}^{\mathrm{S}}$ and $L_{2}^{\mathrm{D}}$ have the same signs. The factor $M / \mu$ also helps the large contributions of these terms, $\left(L_{2}^{\mathrm{S}}+L \frac{\mathrm{D}}{2} / \sqrt{ } 2\right)$, to the total cross section. In the terms $H_{0}^{\mathrm{s}}$ and $H^{\mathrm{ss}}$, in the effective $\pi \mathrm{NN}$ interaction, the contributions of the D-state of the deuteron wave function are almost of the same order as the S-state contributions.

In order to compare the contributions of D-state with those of S-state in the deuteron wave function, we show in fig. 3 the total cross sections obtained separately when only the S-state of the deuteron wave function was considered and when only the D-state was taken into account in the deuteron wave function. 
Table 1

The numerical values of the integrals $L_{0}, L_{1}, L_{2}$. The formulae are given in the text. The quantity $\eta$ is the pion momentum (divided by $\mu c$ )

\begin{tabular}{llllllll}
\hline$\eta$ & $L_{0}^{\mathrm{S}}$ & $L_{0}^{\mathrm{D}}$ & $L_{1}^{\mathrm{S}}$ & $L_{1}^{\mathrm{D}}$ & $L_{2}^{\mathrm{S}}$ & $L_{2}^{\mathrm{D}}$ \\
\hline 0.150 & -0.0956 & 0.147 & -0.0193 & 0.0630 & -0.1184 & -0.0471 \\
0.172 & -0.0950 & 0.147 & -0.0192 & 0.0631 & -0.1183 & -0.0471 \\
0.192 & -0.0944 & 0.146 & -0.0190 & 0.0631 & -0.1182 & -0.0471 \\
0.211 & -0.0938 & 0.146 & -0.0189 & 0.0632 & -0.1181 & -0.0471 \\
0.231 & -0.0927 & 0.146 & -0.0187 & 0.0630 & -0.1178 & -0.0470 \\
0.251 & -0.0919 & 0.146 & -0.0186 & 0.0632 & -0.1176 & -0.0470 \\
0.270 & -0.0912 & 0.146 & -0.0184 & 0.0632 & -0.1174 & -0.0469 \\
0.290 & -0.0901 & 0.145 & -0.0182 & 0.0631 & -0.1171 & -0.0468 \\
0.308 & -0.0890 & 0.145 & -0.0180 & 0.0631 & -0.1168 & -0.0467 \\
0.328 & -0.0880 & 0.145 & -0.0178 & 0.0631 & -0.1165 & -0.0466 \\
0.348 & -0.0868 & 0.144 & -0.0175 & 0.0631 & -0.1162 & -0.0465 \\
0.367 & -0.0857 & 0.144 & -0.0173 & 0.0632 & -0.1159 & -0.0465 \\
0.385 & -0.0847 & 0.144 & -0.0171 & 0.0632 & -0.1156 & -0.0464 \\
\hline
\end{tabular}

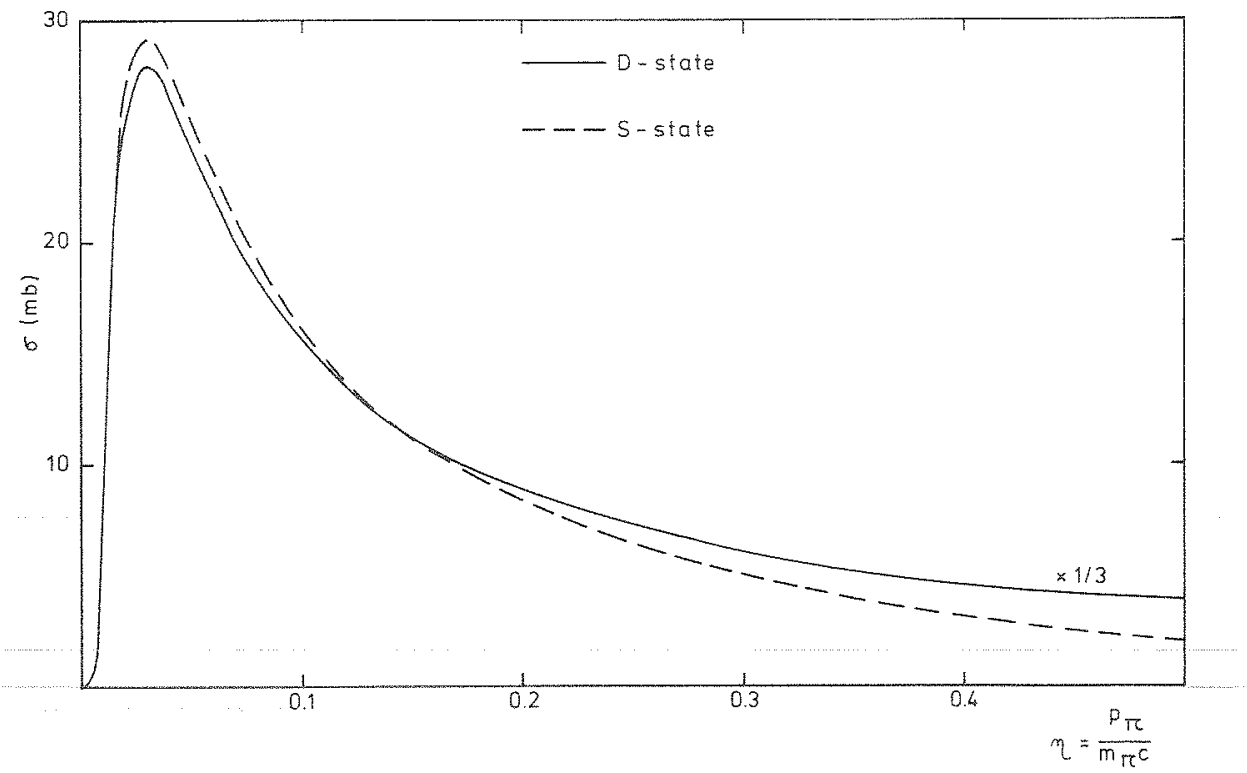

Fig. 3. The total cross sections for the process $\pi^{+}+d \rightarrow p+p$. The solid line is obtained when only the D-state is taken into account in the deuteron wave function. The dotted line corresponds to the case when only the S-state is teken into account in the deuteron wave function. The phase difference is $180^{\circ}$ between the contributions from the $S$ - and $D$-states of the deuteron wave function to the matrix element. 
Generally, in the values of the $\pi \mathrm{N}$ scattering lengths obtained by several people, the set $a_{1}-a_{3}$ are usually close to each other, but the other set $a_{1}+2 a_{3}$ are very different from each other (see e.g. ref. [16]). Therefore, it is also very interesting to determine this quantity by the analysis of the process $\pi^{+}+d \rightarrow p+p$ at very low energy. We obtained $\lambda=0.251 \mathrm{~mm}$. After some simple algebra, this gives the value of scattering length, $a_{1}+2 a_{3}=-0.055$ for the fixed value $a_{1}-a_{3}=0.291$ which was obtained by Samaranayake and Woolcock [15].

We also calculated the inverse process $p+p \rightarrow d+\pi^{+}$at threshold, and obtained

$$
\sigma\left(\mathrm{p}+\mathrm{p} \rightarrow \pi^{+}+\mathrm{d}\right)=211 \eta(\mu \mathrm{b})
$$

except for the Coulomb correction factor. This value is just between those obtained experimentally by Richard-Serre et al. [10] and Rose [13].

\section{The $\pi^{--}$meson absorption by a deuteron from the atomic 1 S orbit}

One of the most important and interesting applications of the effective $\pi \mathrm{NN}$ interaction is the $\pi^{-}$meson absorption by light nuclei from an atomic orbit. It seems to be reliable that the $\pi^{-}$meson in an atomic orbit is absorbed by a nucleon pair in the nucleus followed by two-nucleon emission. Even after the importance of rescattering effects is pointed out $[3,4]$ it is surprising that several authors perform calculations of the absorption rate or the spectrum of the particles emitted, using only the linear, Galilean invariant $\pi N N$ interaction given in eq. (17). This seems to be due to the fact that the formulae given previously were not elegant enough to attract people to overcome the expense of complications. Also, the experimental data include not only large statistical errors but also a scarcity of quantities. However, the rescattering effects are so important that we emphasize the need for the recalculation of old papers. It is already pointed out that a lot of information on the nucleus can be obtained from pion absorption. The actual aim of the proposal of the effective $\pi \mathrm{NN}$ interaction was to construct the basic form of the interaction which is particularly suited for low energy and especially for the stopped $\pi^{-}$meson absorption.

As a first attempt at the application of our effective interaction to the stopped $\pi^{-}$meson absorption problems, it is worthwhile to pick up the deuteron which is the first rucleus. The wave functions of the dueteron and the two emitted neutrons are taken in the same forms as given in eqs. (31) and (32). The wave function of a $\pi^{-}$meson bound in the atomic $1 \mathrm{~S}$ state is

$$
\langle 0|\hat{\phi}| 1\rangle=\frac{2}{a_{0}^{\frac{3}{2}}} \exp \left(-\frac{r}{2 a_{0}}\right) Y_{00}(\hat{r}),
$$


when the center of mass is chosen at the origin. Here, $a_{0}$ is the Bohr radius. Performing some mathematics, we obtain the absoprtion rate

$$
W=\frac{16 \pi g^{2} \hbar^{2} k}{3 a_{0}^{3} M \mu c(2 \pi)^{3}}[F(k)]^{2},
$$

where

$$
\begin{aligned}
F(k) & =\left\{J_{1}^{\mathrm{S}}(k)+\frac{1}{\sqrt{2}} J_{1}^{\mathrm{D}}(k)\right)+\lambda \sqrt{2}\left[\left(J_{2}^{\mathrm{S}}(k)+\frac{1}{\sqrt{2}} J_{2}^{\mathrm{D}}(k)\right)\right. \\
& \left.-\frac{M}{\mu}\left\{J_{3}^{\mathrm{S}}(k)+\frac{1}{\sqrt{2}} J_{3}^{\mathrm{D}}(k)\right)\right] .
\end{aligned}
$$

The quantities $\lambda$ and $\beta$ are defined by the eqs. (35) and (36).

The quantities $J_{1}^{\mathrm{S}}, \ldots J_{3}^{\mathrm{D}}$ are given by the integrals (37) provided the Bessel function $j_{0}\left(\frac{1}{2} k_{0} r\right)$ is replaced by $\exp \left(-r / 2 a_{0}\right)$. The numerical values of the quantities $J_{1}^{\mathrm{S}}, \ldots, J_{\mathrm{S}}^{\mathrm{D}}$, obtained with the Hamada-Johnston potential are tabulated in table 2.

The absorption rate yields

$$
W=1.10 \times 10^{16} \mathrm{sec}^{-1}
$$

with our value $\lambda=0.251 \mathrm{fm}(\lambda=0.1793(\mathrm{~h} / \mu \mathrm{c}))$ which was determined through the process $\pi^{+}+d \rightarrow p+p$ in the previous section. Since the absorption rate is very sensitive to the s-wave scattering length, it is also possible to determine this quantity if the absorption rate is precisely measured. Koltun and Reitan [3] calculated the $\pi^{-}$ absorption rate by a deuteron from the atomic IS orbit using the Hamada-Johnston potential. Their result is $0.84 \times 10^{15} \mathrm{sec}^{-1}$. There is a large discrepancy between the value of the absorption rate obtained by Koltun-Reitan and our result. The numerical values of integrals $J_{i}(k)$ are tabulated in table $2 . J_{1}(k), J_{2}(k)$ and $J_{3}(k)$ are contributions from the terms of $H_{0}^{\mathrm{s}}, H^{\mathrm{sS}}$ and $H^{\mathrm{sp}}$ in the effective $\pi \mathrm{NN}$ interaction, respectively. $J_{1}^{\mathrm{S}}(k), J_{1}^{\mathrm{D}}(k)$ and $J_{3}^{\mathrm{S}}(k)$ have comparatively larger values than the others. As it is seen in eq. (41), $J_{3}^{S}(k)$ actually has a small effect because of the coupling constant $\lambda$ by which $J_{3}^{\mathrm{S}}(k)$ is multiplied. However, $J_{1}^{\mathrm{S}}(k)$ and $J_{1}^{\mathrm{D}}(k)$ have different signs and $\left(J_{1}^{\mathrm{S}}(k)+J_{1}^{\mathrm{D}}(k) / \sqrt{ } 2\right)$ becomes very small by cancellation. This causes a small contribution from the $H_{0}^{S}$ term of effective interaction to the total absorption rate. The theoretical values of the total absorption rate are given in table 3 . For $\lambda=0$, the re-

Table 2

Numerical values of the integrals $J_{1}, J_{2}, J_{3}$ (see text)

\begin{tabular}{cccccc}
\hline$J_{1}^{\mathrm{S}}$ & $J_{1}^{\mathrm{D}}$ & $J_{2}^{\mathrm{S}}$ & $J_{2}^{\mathrm{D}}$ & $J_{3}^{\mathrm{S}}$ & $J_{3}^{\mathrm{D}}$ \\
\hline-0.0964 & 0.1420 & -0.0188 & 0.0608 & -0.1150 & -0.0455 \\
\hline
\end{tabular}


Table 3

Absorption rate of $\pi^{-}$mesons on a deuteron from the atomic $1 \mathrm{~S}$ orbit. The scattering length $a_{1}+2 a_{3}$ which is connected with the coupling constant of the effective $\pi \mathrm{NN}$ interaction is given in units of the pion Compton wave length for $a_{1}-a_{3}=0.291$.

\begin{tabular}{lccc}
\hline$a_{1}+2 a_{3}(\hbar / \mu c)$ & -0.035 & -0.055 & \\
$\lambda(\mathrm{fm})$ & 0.232 & 0.251 & 0 \\
$W\left(\mathrm{sec}^{-1}\right)$ & $0.942 \times 10^{16}$ & $1.10 \times 10^{16}$ & $0.133 \times 10^{14}$ \\
\hline
\end{tabular}

scattering effects are neglected, namely only the direct term $H_{0}^{\mathrm{S}}$ is taken into account. It is easily seen that the rescattering effects raise the value of the total absorption by about $10^{2}$.

\section{Concluding remarks}

First of all, it should be emphasized that the effective $\pi$ NN interaction proposed here includes no free parameter in the sense that it does not contain any phenomenological quantity. Furthermore, our effective Hamiltonian has a form which is convenient to apply to the problems of $\pi^{-}$meson absorption from atomic $S$ - and $\mathrm{P}$. states. It has been shown that our theory produced an excellent agreement with the experimental data of the reaction $\pi^{+}+\mathrm{d} \rightarrow \mathrm{p}+\mathrm{p}$ at low energy.

The coupling constant involved in our theory is connected with the scattering lengths of $\mathrm{S}$-wave $\pi \mathrm{N}$ interactions and its sensitivity is reflected in the total cross section of the process $\pi^{+}+d \leftrightharpoons p+p$ and the stopped $\pi^{-}$meson absorption rate. The values of $\pi N$ scattering lengths determined over ten years are tabulated in ref. [16]. The values of $a_{1}-a_{3}$ are very close to each other while those of $a_{1}+2 a_{3}$ are surprisingly unstable. Most recently, Samaranayake and Woolcock [16] recalculated these quantities by a $\chi^{2}$-fit over the whole energy range up to $2 \mathrm{GeV}$. Their result is $a_{1}+2 a_{3}=0.0031 \pm 0.008$. From the $0.270 \mathrm{MeV}$ data only, they obtained $a_{1}+2 a_{3}=0.0212 \pm 0.0086$, while $a_{1}+2 a_{3}=-0.0543 \pm 0.0111$ from the data above $270 \mathrm{MeV}$. The large differences should be noticed among these values obtained by them. This tells us the difficulty of the determination of this quantity. Then, it may be interesting to try to determine this quantity by a somewhat different method. In order to obtain the value of $a_{1}+2 a_{3}$ through the reaction $\pi^{+}+\mathrm{d} \rightarrow \mathrm{p}$ $+p$ at very low energy (up to $\eta=p_{\pi} / \mu c=0.3$ ), we applied our theory. Our best fit obtained with a $\chi^{2}$ of 12.2 for 14 degrees of freedom is $a_{1}+2 a_{3}=-0.055$ for the fixed value $a_{1}-a_{3}=0.291$ which was previously obtained by Samaranayake and Woolcock [15]. A more precise value for the scattering length $a_{1}+2 a_{3}$ could be obtained when the absorption rate of stopped $\pi^{-}$mesons by a deuteron is measured with great precision, since the deuteron wave function is well known.

In the analysis of the process $\pi^{+}+d \rightarrow p+p$ and the stopped $\pi^{-}$meson absorp- 
tion by deuterium, the following points have been clarified. (1) The contribution of the D-state component of the deuteron wave function to the matrix element is 1.7 times larger than that of the S-state component, but they have opposite signs. (ii) The contributions of the direct term $H_{0}^{\mathrm{S}}$ in the effective $\pi \mathrm{NN}$ interaction to the total cross section and absorption rate are suppressed very much because of the strong cancellation between the S-and D-state contributions in the $H_{0}^{S}$ term, and the rescattering effects become proportionally important.

One of us (I.T.C.) would like to thank Professor J. Humblet and all of the staff for the kind hospitality extended to him at the Institut de Physique, Universite de Liege. It is also acknowledged that this work was done with the financial support of the Institut Interuniversitaire des Sciences Nucléaires, Belgium.

\section{References}

[1] T. Ericson, Phys. Letters 2 (1962) 278; M. Jean, Nuovo Cimento Suppl. 2(1964) 400.

[2] T.I. Kopaleishvili and I.Z. Machabeli, Sov. J. Nucl. Phys. 2 (1966)697; T.l. Kopaleishvili, Sov. J. Nucl. Phys. 1 (1965) 686; J.M. Eisenberg and J. LeTourneux, Nucl. Phys. B3 (1967) 47; D.S. Koltun and A. Reitan, Phys. Rev. 155 (1967) 1139; 1.-T. Cheon, Phys. Rev. 158 (1967) 900; Phys. Letters 268 (1968) 549.

[3] D.S. Koltun and A. Reitan, Phys. Rev. 141 (1966) 1413; T.L. Kopaleishvili, I.Z. Machabeli and M.Sh. Chachkhunashvili, Sov. J. Nucl. Phys. $13(1971) 300$.

[4] 1.T. Cheon and A. Tohsaki, Nucl. Phys. B6 (1968) 586; K. Nishmoto, H. Ohtsubo and H. Narumi, Prog. Theor. Phys. 46 (1971) 135.

[5] L. Foldy and S. Wouthuysen, Phys. Rev. 78 (1950) 29; F. Dyson, Phys. Rev. 73 (1948) 929.

[6] A.F. Woodruff, Phys. Rev, 117 (1960) 1113.

[7] T.H. Field, J.G. Fox, J.A. Kane, R.A. Stallwood and R.B. Sutton, Phys. Rev. 109 (1958) 1704.

[8] C. Lazard, J.L. Ballot and F. Becker, Nuovo Cimento 65B (1970) 117.

[9] G.F. Chew, M.L. Goldberger, F.E. Low and Y. Nambu, Phys. Rev. 106 (1957) 1337.

[10] C. Richard-Serre, W. Hirt, D.F.Measday, E.G. Michaelis, M.J.M. Saltmarsh and P. Skarek, Nucl. Phys. B20 (1970) 413.

[11] D. Schiff and J. Tran Thanh Van, Nucl. Phys. B5 (1968) 529.

[12] H. Myazawa, Phys. Rev. 104 (1956) 1741.

[13] C.M. Rose, Ir., Phys. Rev. 154(1967) 1305.

[14] M.L. Goldberger and K.M. Watson, Collision theory, Wiey, New York, 1965), chap. 10.

[15] V.K. Samaranayake and W.S. Woolcock, Phys. Rev. Letters 15 (1965) 936.

[16] A. Reitan, Nucl. Phys. B11 (1969) 170.

[17] V.K. Samaranayake and W.S. Woolcock, Nuc. Phys. B48 (1972) 205. 
ERPnTYM

I- Tong CHFON and $y$. Cugnon,

Effectve Interaction for pion Ahsorption anc Production bv

pwo rucleons at Low Pnergäes,

Nuc1. phys. B66(1973)42.

A programing excor and a misnint were found in calculation of the stopped $\pi^{-}$arsorption rate by a deuteron. we vant to present here correct ones. Eo. (4O) and Iable 3 of the paper should be read as follows:

$$
\begin{aligned}
& w=\frac{16 g^{2} k^{2} k}{3 a^{3} \mu c}[F(k)]^{2}, \\
& W=0.126 \times 10^{16} \mathrm{sec}^{-1}=
\end{aligned}
$$

\begin{tabular}{cccc}
$a_{1}+2 a_{3}(h / \mu c)$ & -0.035 & -0.055 & \\
$\lambda(\operatorname{mm})$ & 0.232 & 0.251 & 0 \\
$w\left(\sec ^{-1}\right)$ & $0.108 \times 10^{16}$ & $0.126 \times 10^{16}$ & $0.152 \times 10^{12}$ \\
\hline
\end{tabular}


